\title{
BMJ Open Validity and reliability of the Patient Centred Assessment Method for patient complexity and relationship with hospital length of stay: a prospective cohort study
}

Shuhei Yoshida, ${ }^{1,2,3}$ Masato Matsushima, ${ }^{2}$ Hidetaka Wakabayashi, ${ }^{4}$ Rieko Mutai, ${ }^{2}$ Shinichi Murayama, ${ }^{2,5}$ Tetsuro Hayashi, ${ }^{2,6}$ Hiroko Ichikawa, ${ }^{2,7}$ Yuko Nakano, ${ }^{2,8}$ Takamasa Watanabe, ${ }^{1,2,3}$ Yasuki Fujinuma ${ }^{3,9,10}$

To cite: Yoshida S,

Matsushima M, Wakabayashi $\mathrm{H}$, et al. Validity and reliability of the Patient Centred Assessment Method for patient complexity and relationship with hospital length of stay: a prospective cohort study. BMJ Open 2017;7:e016175. doi:10.1136/ bmjopen-2017-016175

- Prepublication history and additional material are available. To view these files please visit the journal online (http://dx.doi. org/10.1136/bmjopen-2017016175).

Received 3 February 2017 Revised 21 March 2017 Accepted 21 March 2017
CrossMark

For numbered affiliations see end of article.

Correspondence to Dr Shuhei Yoshida; yoshida.shuhei.0810@gmail. com

\section{ABSTRACT}

Objectives Several instruments for evaluating patient complexity have been developed from a biopsychosocial perspective. Although relationships between the results obtained by these instruments and the length of stay in hospital have been examined, many instruments are complicated and not easy to use. The Patient Centred Assessment Method (PCAM) is a candidate for practical use. This study aimed to test the validity and reliability of the PCAM and examine the correlations between length of hospital stay and PCAM scores in a regional secondary care hospital in Japan.

Design Prospective cohort study.

Participants and setting Two hundred and one patients admitted to Ouji Coop Hospital between July 2014 and September 2014.

Main predictor PCAM total score in initial phase of hospital admission.

Main outcome Length of stay in hospital.

Results Among 201 patients (Female/Male=98/103) with mean (SD) age of $77.4 \pm 11.9$ years, the mean PCAM score was $25 \pm 7.3$ and mean (SD) length of stay in hospital (LOS) $34.1 \pm 40.9$ days. Using exploratory factor analysis to examine construct validity, PCAM evidently has a two-factor structure, comprising medicine-oriented and patient-oriented complexity. The Spearman rank correlation coefficient for evaluating criterion-based validity between PCAM and INTERMED was 0.90 . For reliability, Cronbach's alpha was 0.85 . According to negative binomial regression analyses, PCAM scores are a statistically significant predictor $(p<0.001)$ of LOS after adjusting for age, gender, Mini Nutritional Assessment Short-Form, Charlson Comorbidity Index, serum sodium concentration, total number of medications and whether public assistance was required. In another model, each factor in PCAM was independently correlated with length of stay in hospital after adjustment (medicine-oriented complexity: $p=0.001$, patient-oriented complexity: $\mathrm{p}=0.014$ ).

Conclusion PCAM is a reliable and valid measurement of patient complexity and PCAM scores have a significant correlation with hospital length of stay.
Strengths and limitations of this study

- This study examined the validity and reliability of Patient Centred Assessment Method (PCAM), which was a practical tool to evaluate patients comprehensively from a biopsychosocial perspective.

- PCAM had the relation to an important clinical outcome not only in primary care but also in secondary care; length of stay in hospital.

- Inter-rater variability of PCAM scores was neither evaluated in this study nor was the spectrum of diseases on admission to community-based hospitals taken into account.

- Differences in care setting, type and severity of disease, insurance systems and other factors may have an effect on PCAM scores.

\section{INTRODUCTION}

Shortening the length of stay in hospital (LOS) leads to improving the quality of care. $^{12}$ Organisation for Economic Co-operation and Development Health Statistics 2015 indicate that the average LOS in Japan is 17.2 days, which is the longest of the 34 countries surveyed. ${ }^{3}$

Various factors relating to LOS have been identified from a biopsychosocial perspective; namely, the biomedical factors of comorbidity, ${ }^{4}$ electrolyte imbalance, ${ }^{5}$ malnutrition ${ }^{6}$ and deconditioning ${ }^{7}$; the psychological factors of dementia, ${ }^{8}$ depression ${ }^{9}$ and anxiety ${ }^{10}$; and the social factors of cohabitation with family, ${ }^{11}$ caregiver, $^{12}$ marital status ${ }^{13}$ and insurance. ${ }^{14}$ Several comprehensive scales have been developed to evaluate the biopsychosocial aspects of patient complexity. Among them, INTERMED and Oxford Case Complexity Assessment 
Measure (OCCAM) scores are reportedly correlated with LOS. $^{15-19}$

The Patient Centred Assessment Method (PCAM) ${ }^{20}$ is an advanced version of the Minnesota complexity assessment method (MCAM), ${ }^{21}$ which originated from INTERMED, a tool for practical use in the primary care setting that was developed in Scotland. PCAM comprises 12 items in contrast with the 20 items of INTERMED. Although, the validity and reliability of INTERMED have been evaluated in secondary care settings, it is relatively impractical because it takes long time to answer many times and also it can be applied only to secondary care setting. In contrast, PCAM may be more appropriate for daily work in a primary care setting as it has fewer items and simpler questions than INTERMED. However, it is unclear whether PCAM can be applied in secondary care settings.

Although, the patients receiving primary care often need unscheduled secondary care (ie, hospital admission), the requisite data, especially those concerning psychosocial factors, cannot be transmitted smoothly. Assessing PCAM at time of admission, that is, during the transition from a primary to a secondary care setting, made the patient complexity come to the front. This accumulation can affect aspects of the patient's course during the hospital admission, such as LOS. The aims of this study were to evaluate the validity and reliability of PCAM and examine the relationship between PCAM and LOS in an acute care unit in a secondary care setting in an urban neighbourhood of Tokyo, Japan.

\section{METHODS}

\section{Study group and setting}

This was a prospective cohort study. The subjects were all inpatients who were admitted to the acute care unit of Ouji Coop Hospital between 1 July 2014 and 30 September 2014. Ouji Coop Hospital is a regional secondary care hospital and family physician teaching facility with 159 beds in Kita-ku, Tokyo, Japan, which is a district with a high population ageing rate approximately $15 \mathrm{~km}$ north of central Tokyo. Most inpatients require management of medical conditions: pneumonia, urinary tract infection, acute exacerbation of chronic illness (chronic obstructive pulmonary disease, diabetes, etc.), dermatologic conditions such as decubitus ulcers and cellulitis, orthopaedic conditions such as lumbar compression and proximal femoral fractures that do not require surgery and cancer pain, that is not being controlled in ambulatory or home medical care. Because this hospital has no surgical facilities, patients who need surgical treatment are referred to other hospitals. Exclusion criteria were: age younger than 20 years, refusal to participate in the study, and length of stay fixed at time of admission (eg, for colonic polypectomy).

\section{Measurement variables and evaluation process}

Outcome measure: LOS

LOS was automatically calculated by the electronic medical record system for all participants. ${ }^{22}$
Predictor variables and covariates

1) Complexity scales

One of the authors (SY) evaluated PCAM and INTERMED by interviewing patients and patients' family members independently from other doctors. The Japanese version was used for INTERMED. ${ }^{23}$ INTERMED and PCAM scores were evaluated simultaneously. The PCAM was not available in Japanese, so the interviewer made a translation and asked the questions in Japanese. The interviewer was able to judge the items on the PCAM and completed the assessment form by considering the items in a Japanese context. The PCAM guide included sample questions for each item, and the interviewer was able to employ appropriate questions when translating into Japanese. For ethical reasons, staff members were informed that the researchers could provide them with the results of these complexity scores if asked. No-one requested these results during the research period.

\section{PCAM}

MCAM originated from INTERMED, was designed for action-based evaluation of complexity in primary care settings, and has been used mainly in educational settings. PCAM, which was developed from MCAM by Pratt et al. ${ }^{20}$, evaluates patients' centeredness in addition to their experience and is intended for use with patients with comorbid conditions or multimorbidity. It has been suggested that PCAM assists medical staff to refer patients to non-medical services dealing with psychosocial needs and that evaluation of PCAM by nurses improves their understanding of each patient's complexity.

\section{INTERMED}

INTEMED, which was developed by Huyse et al. ${ }^{1617}$ in the Netherlands, evaluates patient complexity from a biopsychosocial perspective and assesses health service needs. It has been INTERMED translated into various languages; a Japanese version was developed by Kishi et al. ${ }^{23}$ and its reliability and validity have been confirmed.

2) Participants' characteristics

Age, gender, number of medications, and serum sodium concentrations were obtained from electronic medical records on admission. Ancillary staff members administered the Charlson Comorbidity Index (CCI) ${ }^{24}$ and Mini Nutritional Assessment Short-Form (MNA-SF) ${ }^{25-27}$ and asked patients or family members about the number of family members living with the patient, whether they had a principal care giver, and whether they had received public assistance.

\section{$\mathrm{CCl}$}

The CGI was developed in 1987 and evaluates comorbidities. It checks the following medical conditions and the resultant scores are summed: myocardial infarction, congestive heart failure, peripheral vascular disease, cerebrovascular disease, dementia, chronic obstructive pulmonary disease connective tissue disease, peptic ulcer, diabetes mellitus, moderate to severe chronic kidney 
disease, hemiplegia, leukaemia, malignant lymphoma, solid tumour, liver disease, and AIDS. In 2014 a correlation between CCI score and mortality post-admission to acute hospitals (3 months, 1 year and 5 years) was reported ${ }^{4}$.

\section{MNA-SF}

Nutritional status was assessed by the Mini Nutritional Assessment Short Form (MNA-SF). The MNA-SF comprises six questions addressing: 1) changes in food intake over the past 3 months, 2) weight loss over the past 3 months, 3) mobility, 4) psychological stress or acute disease in the past 3 months, 5) neuropsychological problems, and 6) body mass index.

\section{Analysis and statistical methods}

The criterion-based validity of PCAM was evaluated by using Spearman's rank correlation coefficient between PCAM and INTERMED. Exploratory factor analysis was conducted to examine PCAM's construct validity. In the process of factor analysis, factors with eigenvalues $\geq 1$ were adopted and factor loading was rotated using promax rotation to interpret the factors. Factor loadings that were $\geq 0.3$ were adopted and those factors interpreted. Cronbach's alpha, an indicator of internal consistency, was calculated to examine PCAM's reliability.

The association between LOS and PCAM was evaluated by negative binomial regression analyses in four models. In model 1, the predictive variable was total PCAM score and covariates were age and gender. In model 2 , the predictive variable was total PCAM score and covariates were age, gender, MNA-SF, CCI scores, serum sodium concentration, total number of medications and whether the patient required public assistance. In model 3, the predictive variables were the factors derived from factor analysis of PCAM and covariates age and gender. In model 4 , the predictive variables were the factors derived from factor analysis of PCAM and covariates age, gender, MNA-SF,CCI scores, serum sodium concentration, total number of medications and whether the patient required public assistance.

To test the assumption of negative binomial regression, binomial proportions with Clopper-Pearson exact 95\% CI for each discharge were first calculated. ${ }^{28}{ }^{29}$ The relationship between binomial proportions with 95\% CIs and discharge day was examined whether the independence assumption of attempts with a common underlying probability of discharge was violated. Second, the negative binomial regression model was assumed to have the conditional means, which was not equal to the conditional variances. Therefore, the null hypothesis that the overdispersion parameters of models were 0 was examined by likelihood ratio tests.

To check multicollinearity, the correlation among predictors were calculated by the pairwise Pearson's correlation coefficient and variance inflation factors.

All statistical analyses were performed using STATA/ SE version $12 .{ }^{30}$ In negative binomial regression models,
$\mathrm{P}<0.025$ was considered statistically significant because each of PCAM and two factors of PCAM was examined twice by negative binomial regression. Otherwise, $p<0.05$ was considered statistically significant.

\section{Ethical considerations}

The Ethics Committee at Tokyo Hokuto Health Co-operative approved the present protocol. The approval number was 70 . For informed consent, we verbally explained the study and we gave the patients a document, which clarified the study and provided details such as privacy protection. We also stated on the questionnaires asking about the patients' background information that by answering the questions, the patients were deemed to have agreed to participate in the study.

After receiving the completed questionnaire, SY interviewed to evaluate PCAM and INTERMED.

\section{RESULTS}

Among 263 admissions, 46 patients met the exclusion criteria: 1 person refused to participate in the study and 45 patients had a pre-determined, fixed LOS on admission. In addition, 2 patients could not be interviewed owing to cognitive impairment, and there was no possibility of interviewing their family; 4 individuals were immediately referred to other hospitals owing to emergency operations; 10 patients had no score for the MNA-SF owing to missing values. Thus, 201 inpatients participated in this study. Their characteristics are shown in table 1.

Table 2 showed classification of main diagnosed diseases of participants.

Total PCAM and INTERMED scores were distributed as shown in figure 1. The mean (SD) PCAM score was $25 \pm 7.3$ and mean (SD) INTERMED score was 22.8 \pm 9.7 . No floor or ceiling effects were identified. The correlation between PCAM and INTERMED is shown in figure 2. Spearman's rank correlation coefficient was 0.90 . Factor analysis of PCAM is shown in table 3. Two factors of eigenvalue $\geq 1$ were identified. Factor loading was rotated using promax rotation to interpret the factors. Factor loadings $\geq 0.3$ were selected and interpreted. The items 'Health and Well-being': 2, 3 and 4, 'Social Environment':

\begin{tabular}{|c|c|c|}
\hline Age, mean (SD), years & 77.4 & (11.9) \\
\hline Female, n (\%) & 98 & $(48.8)$ \\
\hline No. of medications, mean (SD) & 6.4 & $(4.1)$ \\
\hline Cancer, n (\%) & 36 & (17.9) \\
\hline Diabetes mellitus, n (\%) & 44 & (21.9) \\
\hline Receiving public assistance, $n(\%)$ & 38 & (18.9) \\
\hline $\begin{array}{l}\text { Charlson Comorbidity Index score, mean } \\
\text { (SD) }\end{array}$ & 2.0 & (2.2) \\
\hline Serum sodium, mean (SD), mEq/L & 138 & (5.6) \\
\hline $\begin{array}{l}\text { Mini Nutritional Assessment Short-Form } \\
\text { score, mean (SD) }\end{array}$ & 7.9 & (3.8) \\
\hline
\end{tabular}


Table 2 Main diseases diagnosed among participants

\begin{tabular}{lrl}
\hline Classification of main diseases & N & Per cent \\
\hline Neoplasms & & \\
\hline Total & 29 & 14.4 \\
Stomach & 6 & \\
Colon & 4 & \\
Lung & 6 & \\
\hline
\end{tabular}

Endocrine, nutritional and metabolic diseases

$\begin{array}{lcc}\text { Total } & 17 & 8.5 \\ \text { Diabetes mellitus } & 13 & \\ \text { Mental and behavioural disorders } & & \\ \text { Total } & 6 & 3.0 \\ \text { Alzheimer disease } & 5 & \end{array}$

Diseases of the nervous system

$\begin{array}{lll}\text { Total } & 7 & 3.5\end{array}$

Diseases of the circulatory system

$\begin{array}{lcc}\text { Total } & 28 & 13.9 \\ \text { Heart failure } & 11 & \\ \text { Cerebral infarction } & 10 & \\ \text { Diseases of the respiratory system } & & \\ \text { Total } & 45 & 22.4 \\ \text { Pneumonia } & 29 & \\ \text { Asthma } & 7 & \\ \text { Emphysema } & 2 & \\ \text { Diseases of the digestive system } & & \\ \text { Total } & 29 & 14.4 \\ \text { Viral enteritis } & 7 & \\ \text { Cirrhosis of liver } & 4 & \\ \text { Diseases of the skin and subcutaneous tissue } & & \\ \text { Total } & 9 & 4.5 \\ \text { Cellulitis } & 6 & \end{array}$

Diseases of the musculoskeletal system and connective tissue

\begin{tabular}{lrr} 
Total & 19 & 9.5 \\
\hline Fracture & 10 & \\
Diseases of the genitourinary system & & \\
Total & 7 & 3.5 \\
Urinary tract infection & 4 & \\
\hline Others & 5 & 2.5 \\
Total & 201 & 100 \\
\hline
\end{tabular}

2 and 3, and 'Health Literacy and Communication': 1 and 2 contributed to the first factor, which is, thus, composed of patient's internal factors such as mental condition and literacy; it was labelled as patient-oriented complexity. For example, the item with the highest and the second highest factor loading were 'Health Literacy and Communication 1: How well does the client now understand their health and well-being (symptoms, signs or risk factors)
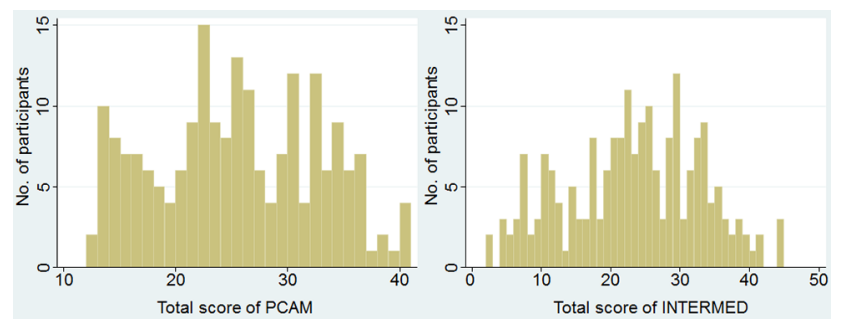

Figure 1 Distribution: total score of Patient Centred Assessment Method (PCAM) and INTERMED.

and what they need to do to manage their health?' and 'Health Literacy and Communication 2: How well do you think your client can engage in healthcare discussions? (Barriers include language, deafness, aphasia, alcohol or drug problems, learning difficulties, concentration)", respectively (cited from PCAM Online ${ }^{31}$ ).

The items 'Health and Well-being': 1, 'Social Environment': 1 and 4, and 'Service Coordination': 1 and 2 contributed to the second factor, which is thus composed of patient's external factors such as care environment and service. Factor 2 was labelled as medicine-oriented complexity. For example, the items with the highest and the second highest factor loading were; 'Service Coordination 1: Do other services need to be involved to help this client?' and 'Service Coordination 2: Are services involved with this client well coordinated?", respectively (cited from PCAM Online ${ }^{31}$ ).

Cronbach's alpha of PCAM, which indicates internal consistency was 0.85 . This level of Cronbach's alpha means internal consistency is acceptable (Cronbach's alpha $>0.80){ }^{32}$

Table 4 shows the result of negative binomial regression analyses, which examined the associations between PCAM and LOS after adjusting the covariates. Factor analysis of PCAM identified two factors, which were labelled as patient-oriented complexity and medicine-oriented complexity. These were used as covariates in models 3 and 4. In models 1 and 2, PCAM was significantly associated with LOS, whereas in models 3 and 4 , both patient-oriented and medicine-oriented factors

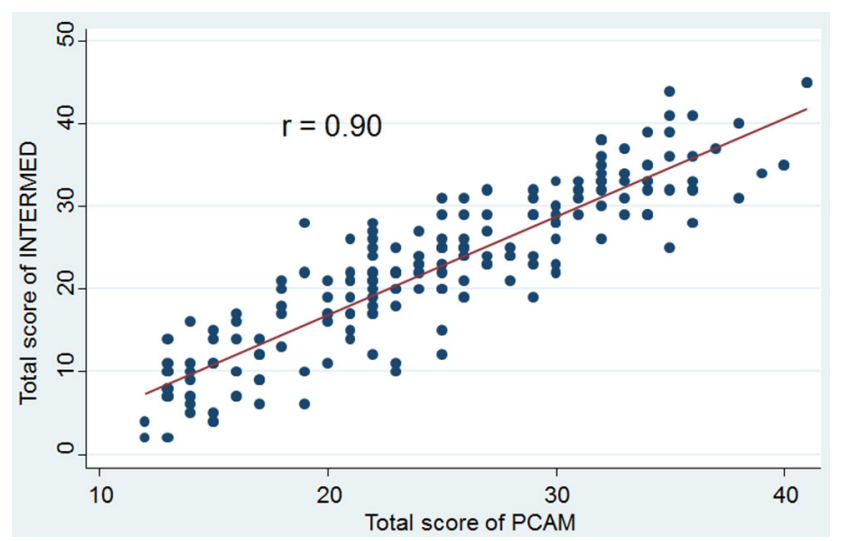

Figure 2 Correlation between Patient Centred Assessment Method (PCAM) and INTERMED. 
Table 3 Factor analysis of the Patient Centred Assessment Method (PCAM)

\begin{tabular}{ccc}
\hline & \multicolumn{2}{l}{ Factor loading } \\
\cline { 2 - 3 } PCAM item & First factor & Second factor \\
\hline Health and well-being & \\
\hline 1 & 0.1202 & 0.3487 \\
2 & 0.3532 & 0.1878 \\
3 & 0.3786 & -0.0521 \\
4 & 0.3857 & 0.0117 \\
\hline Social environment & & \\
\hline 1 & 0.0631 & 0.6859 \\
\hline 2 & 0.4699 & 0.1131 \\
\hline 3 & 0.6152 & 0.1293 \\
4 & 0.0581 & 0.3508 \\
\hline Health literacy and communication & \\
\hline 1 & 0.9914 & -0.1577 \\
\hline 2 & 0.8616 & 0.0140 \\
\hline Service coordination & \\
\hline 1 & -0.1130 & 0.9842 \\
\hline 2 & -0.0531 & 0.9427 \\
\hline
\end{tabular}

were significantly and independently correlated with LOS. Assumptions of negative binomial regression were checked. To test the first assumption, binomial proportions with Clopper-Pearson exact 95\% CI for each discharge were calculated. Figure 3 shows the relationship between binomial proportions with $95 \%$ CIs and discharge day to examine whether the independence assumption of attempts with a common underlying probability of discharge was violated. The interval from $2.84 \%$ to $7.09 \%$ was overlapped in every CI. Therefore, this assumption was not violated. Second, the negative binomial regression model is assumed to have the conditional means which are not equal to the conditional variances. It was examined whether the overdispersion parameters of four models in our manuscript were 0 by likelihood ratio tests. As a result for our 4 models, null hypothesis that the overdispersion parameters were 0 were rejected. Therefore, negative binomial regression models were more appropriate than Poisson models. Other than the pairwise Pearson's correlation coefficient (0.56) between Patient-oriented complexity and Medicine oriented complexity, those among predictors ranged between -0.45 and 0.29 , which indicates that pairwise correlations did not have strong impacts on regression models. In addition to pairwise correlations, variance inflation factors were examined whether there were the multicollinearity among predictor variables. Since the variance inflation factors of each variable in our 4 models ranged between 1.05 and 1.67, no multicollinearity exists.

\section{DISCUSSION}

In the current study, assessment of criterion-based validity in comparison with INTERMED, construct validity by exploratory factor analysis, and reliability by Cronbach's alpha showed that PCAM is a valid and reliable scale in the initial phase of admission to a secondary care hospital. Additionally, total PCAM score on admission correlated significantly with LOS. Moreover, each of two factors derived from factor analysis of PCAM-"patient-oriented complexity' and 'medicine-oriented complexity'-was significantly independently correlated with LOS.

The validity and reliability of PCAM scores were examined. To assess criterion-based validity, INTERMED was used as an external criterion. Spearman's rank correlation coefficient between these scores was 0.90 , which indicates a strong correlation. Thus, PCAM has the potential to substitute for INTERMED. When OCCAM was developed, the Spearman's rank correlation coefficient for OCCAM versus INTERMED scores was assessed and found to be $0.694 .{ }^{19}$ Thus, PCAM scores correlate more strongly with INTERMED scores than do OCCAM scores. The reason for the weaker correlation between OCCAM and INTERMED scores may be that OCCAM includes new elements specifically related to neurological rehabilitation such as excretory and sensory function; however, both OCCAM and PCAM were derived from INTERMED.

Exploratory factor analysis was conducted to examine construct validity and resulted in the identification of two factors. The extracted two factors were patient-oriented complexity, which includes internal patient factors such as mental well-being and literacy, and medicine-oriented complexity, which includes external factors such as social health service and physical health needs. This finding is in accordance with the aim of developing PCAM, as described earlier in the Discussion section. Because other scores measuring complexity were not evaluated by factor analysis, it was not possible to compare this result with other scores. It is uncertain whether these two factors are ideal constructs in a complexity scale.

Cronbach's alpha was 0.85 over 0.80 . Thus, despite the small number of questions, the reliability of PCAM was proved. The test-retest method was not used because patients' conditions changed remarkably during the interval between tests. The parallel test method was also not used, because of the difficulty in ensuring the quality of evaluation.

Negative binomial regression showed that PCAM scores on admission correlated with LOS. Negative binomial regression rather than multiple linear regression was chosen because the distribution of LOS was skewed to the right, as shown in figure 4, and the higher the total PCAM score, the greater was the variability in LOS, as shown in figure 5 .

Other studies have found an association between patient complexity and LOS. A previous study of INTERMED by hierarchical cluster analysis showed that patients with high biopsychosocial complexity 
Table 4 Results of negative binomial regression analyses

\begin{tabular}{|c|c|c|c|c|}
\hline \multirow[b]{2}{*}{ Predictive variable } & \multirow[b]{2}{*}{ Coefficient } & \multicolumn{2}{|l|}{$95 \% \mathrm{Cl}$} & \multirow[b]{2}{*}{$\mathbf{p}$} \\
\hline & & lower & upper & \\
\hline \multicolumn{5}{|l|}{ Model 1} \\
\hline PCAM & 0.054 & 0.038 & 0.070 & 0.001 \\
\hline Age & 0.0098 & -0.000082 & 0.020 & 0.052 \\
\hline Gender & -0.19 & -0.43 & 0.045 & 0.11 \\
\hline \multicolumn{5}{|l|}{ Model 2} \\
\hline PCAM & 0.055 & 0.036 & 0.074 & 0.001 \\
\hline Age & 0.0094 & -0.0014 & 0.020 & 0.088 \\
\hline Gender & -0.20 & -0.45 & 0.048 & 0.1 \\
\hline Mini Nutritional Assessment Short-Form & -0.000059 & -0.039 & 0.039 & 1.00 \\
\hline Charlson Comorbidity Index & 0.000096 & -0.061 & 0.062 & 1.00 \\
\hline Serum sodium concentration & 0.0096 & -0.012 & 0.032 & 0.39 \\
\hline No. of medications & -0.011 & -0.044 & 0.021 & 0.50 \\
\hline Requiring public assistance & 0.089 & -0.21 & 0.39 & 0.57 \\
\hline \multicolumn{5}{|l|}{ Model 3} \\
\hline Medicine-oriented complexity & 0.076 & 0.031 & 0.12 & 0.001 \\
\hline Patient-oriented complexity & 0.041 & 0.012 & 0.071 & 0.006 \\
\hline Age & 0.0090 & -0.00094 & 0.019 & 0.076 \\
\hline Gender & -0.21 & -0.45 & 0.029 & 0.085 \\
\hline \multicolumn{5}{|l|}{ Model 4} \\
\hline Medicine-oriented complexity & 0.083 & 0.035 & 0.13 & 0.001 \\
\hline Patient-oriented complexity & 0.039 & 0.0080 & 0.071 & 0.014 \\
\hline Age & 0.0083 & -0.0026 & 0.019 & 0.14 \\
\hline Gender & -0.23 & -0.48 & 0.023 & 0.075 \\
\hline Mini Nutritional Assessment Short-Form & -0.0016 & -0.041 & 0.038 & 0.94 \\
\hline Charlson Comorbidity Index & -0.0075 & -0.070 & 0.055 & 0.81 \\
\hline Serum sodium concentration & 0.010 & -0.012 & 0.032 & 0.36 \\
\hline No. of medications & -0.0092 & -0.042 & 0.023 & 0.58 \\
\hline Requiring public assistance & 0.12 & -0.19 & 0.43 & 0.44 \\
\hline
\end{tabular}

PCAM, Patient Centred Assessment Method.

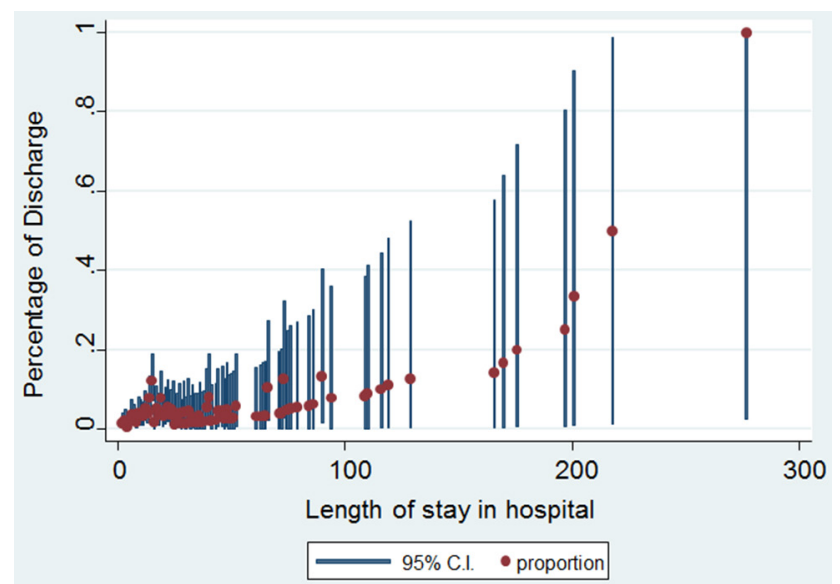

Figure 3 Relationship between binomial proportions with 95\% Cls for each discharge. had longer LOS than those with low biopsychosocial complexity and uncomplicated chronic somatic illness. In a study using OCCAM $^{19}$ patients with $\mathrm{LOS} \geq 81$ had higher total OCCAM scores than those with LOS $<81$. This study showed similar results, even though those studies were conducted in different countries. Thus, even with different cultures and medical administration systems, it is likely that biopsychosocial complexity has an effect on LOS. Moreover, the previous studies used only univariate analysis and thus did not consider confounding factors. Recognising patients with high complexity on admission can facilitate relevant interventions, possibly shortening LOS. PCAM is a candidate tool for evaluating patient complexity and shortening the LOS of patients with high complexity.

In this study, only one researcher evaluated PCAM scores whereas in clinical situations various care providers would evaluate PCAM scores. Inter-rater 


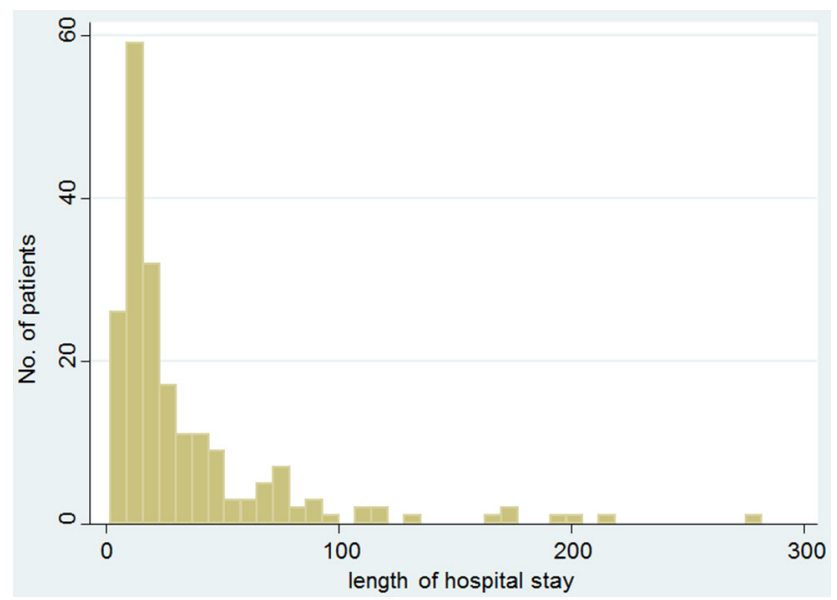

Figure 4 The distribution of length of stay in hospital(LOS), which looks skewed to the right. The mean (SD) LOS was $34.1 \pm 40.9$ days. One hundred and thirty-five participants $(67.2 \%)$ were discharged within 30 days of admission to hospital.

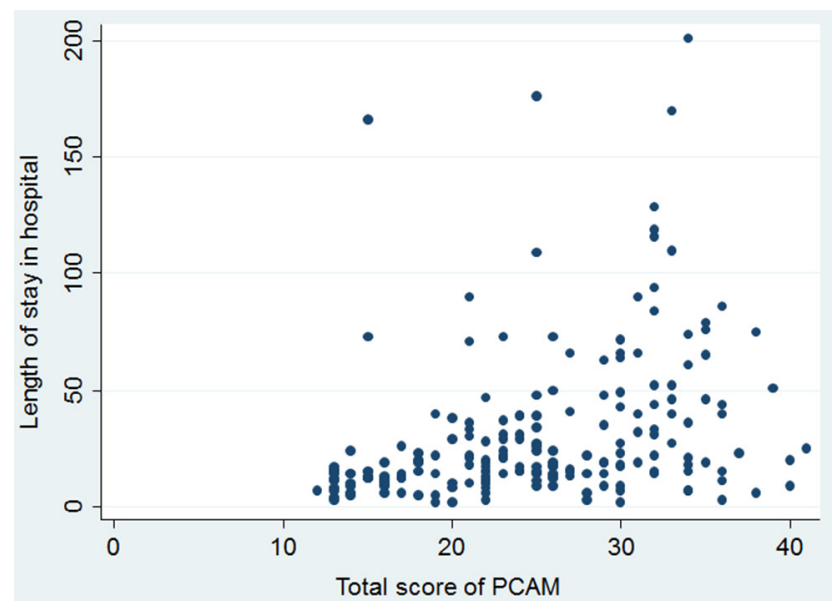

Figure 5 The relationship between total PatientCentred Assessment Method (PCAM) scores and length of stay in hospital(LOS). The higher the total PCAM score, the wider was the variability in LOS.

variability of PCAM scores was neither evaluated in the current study, nor was the spectrum of diseases on admission to community-based hospitals taken into account. Differences in care setting, type and severity of disease, insurance systems and other factors may have an effect on PCAM scores.

\section{FUTURE RESEARCH}

To facilitate widespread use of PCAM as a complexity assessment tool in Japan, we plan to develop a Japanese version of PCAM. Future research is necessary to examine whether identifying problems that need intervention leads to any solution.

\section{CONCLUSION}

Our findings indicate that PCAM is a valid and reliable scale for assessing patient complexity in the initial phase of admission to a secondary care hospital. Moreover, we found that PCAM on admission correlates with LOS.

Author affiliations

${ }^{1}$ Kitaadachi-seikyo Clinic, Japanese Health and Welfare Co-operative Federation, Tokyo, Japan

${ }^{2}$ Division of Clinical Epidemiology, Research Center for Medical Sciences, Jikei University School of Medicine, Tokyo, Japan

${ }^{3}$ Centre for Family Medicine Development, Japanese Health and Welfare Cooperative Federation, Tokyo, Japan

${ }^{4}$ Department of Rehabilitation Medicine, Yokohama City University Medical Center, Yokohama, Japan

${ }^{5}$ Shioiri-seikyo clinic, Japanese Health and Welfare Co-operative Federation, Tokyo, Japan

${ }^{6}$ National Hospital Organization Tokyo Medical Center, Tokyo, Japan

${ }^{7}$ Tokyo Rinkai Byoin, Edogawa-ku, Tokyo, Japan

${ }^{8}$ Japan Small Animal Cancer Center, Saitama, Japan

${ }^{9}$ Seikyo-ukima clinic, Japanese Health and Welfare Co-operative Federation, Tokyo, Japan

${ }^{10}$ Interprofessional Education Research Center (IPERC), Graduate School of Nursing, Chiba University, Chiba, Japan

Acknowledgements The authors would like to thank Rebekah Pratt at Department of Family Medicine and Community Health, University of Minnesota for allowing the use of PCAM.

Contributors SY reviewed and edited the manuscript, conducted the research study and was primarily responsible for data collection, integrity, and analysis. MM reviewed and edited the manuscript and was primarily responsible for data collection, integrity, and analysis. HW, RM, SM, TH, HI, YN, TW and YF reviewed and edited the manuscript, contributed to the discussion of the data and performed critical review of the manuscript. All authors contributed the conception of this work. All authors revised it critically for important intellectual content. All authors approved the final version for publication. All authors agree to be accountable for all aspects of the work. SY is the guarantor of this work and, as such, has full access to all the data in the study and takes responsibility for the integrity of the data and the accuracy of the data analysis.

Competing interests SY worked at Ouji Coop Hospital. YF, SM and TW work as part-time physicians at Ouji Coop Hospital. MM is an adviser of Centre for Family Medicine Development practice-based research network. MM received lecture fee and travel fee for lecture from Centre for Family Medicine Development. MM received the grant from Japan Society for the Promotion of Science. YN received the grant from Japan Society for the Promotion of Science. SY is a resident in family medicine of Centre for Family Medicine Development. TW was a former resident in family medicine of Centre for Family Medicine Development. MM is a Program Director of Jikei Clinical Research Program for Primary-care. YF is a lecturer of Jikei Clinical Research Program for Primary-care. SY, HW, SM, TH, HI and TW were former trainees of Jikei Clinical Research Program for Primarycare. This work was supported by a research grant of fiscal year 2015 from The Jikei University School of Medicine and Postgraduate Medical School. There is no potential competing interest to be declared relevant to this work other than the above description.

Patient consent For informed consent, we verbally explained the study and we gave the patients a document, which clarified the study and provided details such as privacy protection. We also stated on the questionnaires asking about the patients' background information that by answering the questions, the patients were deemed to have agreed to participate in the study.

Ethics approval Tokyo Hokuto Health Co-operative.

Provenance and peer review Not commissioned; externally peer reviewed.

Data sharing statement The database is available only for members of the research team.

Open Access This is an Open Access article distributed in accordance with the Creative Commons Attribution Non Commercial (CC BY-NC 4.0) license, which permits others to distribute, remix, adapt, build upon this work non-commercially, and license their derivative works on different terms, provided the original work is properly cited and the use is non-commercial. See: http://creativecommons.org/ licenses/by-nc/4.0/ 
(c) Article author(s) (or their employer(s) unless otherwise stated in the text of the article) 2017. All rights reserved. No commercial use is permitted unless otherwise expressly granted.

\section{REFERENCES}

1. Fearon $P$, Langhorne P. Services for reducing duration of hospital care for acute stroke patients. Cochrane Database Syst Rev 2012;9:Cd000443.

2. Kondo A, Zierler BK, Isokawa Y, et al. Comparison of outcomes and costs after hip fracture surgery in three hospitals that have different care systems in Japan. Health Policy 2009;91:204-10.

3. OECD. Average length of stay in hospitals. OECD Publishing.

4. Frenkel WJ, Jongerius EJ, Mandjes-van Uitert MJ, et al. Validation of the Charlson Comorbidity Index in acutely hospitalized elderly adults: a prospective cohort study. J Am Geriatr Soc 2014;62:342-6.

5. Wald R, Jaber BL, Price LL, et al. Impact of hospital-associated hyponatremia on selected outcomes. Arch Intern Med 2010;170:294-302

6. Felder S, Lechtenboehmer C, Bally $\mathrm{M}$, et al. Association of nutritional risk and adverse medical outcomes across different medical inpatient populations. Nutrition 2015;31(11-12):1385-93.

7. Wakabayashi $\mathrm{H}$, Sashika $\mathrm{H}$. Malnutrition is associated with poor rehabilitation outcome in elderly inpatients with hospital-associated deconditioning a prospective cohort study. J Rehabil Med 2014:46:277-82.

8. Guijarro R, San Román CM, Gómez-Huelgas R, et al. Impact of dementia on hospitalization. Neuroepidemiology 2010;35:101-8.

9. Verbosky LA, Franco KN, Zrull JP. The relationship between depression and length of stay in the general hospital patient. $J$ Clin Psychiatry 1993;54:177-81.

10. Saravay SM, Steinberg MD, Weinschel B, et al. Psychologica comorbidity and length of stay in the general hospital. Am J Psychiatry 1991;148:324-9.

11. Ismail Z, Arenovich T, Grieve C, et al. Predicting hospital length of stay for geriatric patients with mood disorders. Can J Psychiatry 2012;57:696-703.

12. Mochari-Greenberger H, Mosca M, Aggarwal B, et al. Caregiver status: a simple marker to identify cardiac surgery patients at risk for longer postoperative length of stay, rehospitalization, or death. $J$ Cardiovasc Nurs 2014;29:12-19.

13. Gordon HS, Rosenthal GE. Impact of marital status on outcomes in hospitalized patients. evidence from an academic medical center. Arch Intern Med 1995;155:2465-71.

14. Golabi P, Otgonsuren M, Suen W, et al. Predictors of Inpatient Mortality and Resource utilisation for the Elderly Patients with Chronic Hepatitis $\mathrm{C}(\mathrm{CH}-\mathrm{C})$ in the United States. Medicine 2016;95:e2482.
15. Stiefel FC, de Jonge P, Huyse FJ, et al. INTERMED--an assessment and classification system for case complexity. results in patients with low back pain. Spine 1999;24:378-84.

16. Huyse FJ, Lyons JS, Stiefel FC, et al. "INTERMED": a method to assess health service needs. I. Development and reliability. Gen Hosp Psychiatry 1999;21:39-48.

17. Stiefel FC, de Jonge P, Huyse FJ, et al. "INTERMED": a method to assess health service needs. II. Results on its validity and clinical use. Gen Hosp Psychiatry 1999;21:49-56.

18. de Jonge P, Bauer I, Huyse FJ, et al. Medical inpatients at risk of Extended Hospital Stay and Poor Discharge Health Status. Psychosom Med 2003;65:534-41.

19. Troigros $\mathrm{O}$, Béjot $\mathrm{Y}$, Rodriguez $\mathrm{PM}$, et al. Measuring complexity in neurological rehabilitation: the Oxford Case Complexity Assessment measure (OCCAM). Clin Rehabil 2014;28:499-507.

20. Pratt R, Hibberd C, Cameron I, et al. The patient centered Assessment Method (PCAM): integrating the social dimensions of health into primary care. J Comorb 2015;5;110-9.

21. Macdonald S, Morrison J, Maxwell M, et al. 'A coal face option': GPs' perspectives on the rise in antidepressant prescribing. $\mathrm{Br} J \mathrm{Gen}$ Pract 2009;59:299-307.

22. Sarkies MN, Bowles KA, Skinner EH, et al. Data collection methods in health services research: hospital length of stay and discharge destination. App/ Clin Inform 2015;6:96-109.

23. Kishi $Y$, Matsuki M, Mizushima $\mathrm{H}$, et al. The INTERMED Japanese version: inter-rater reliability and internal consistency. J Psychosom Res 2010;69:583-6.

24. Charlson ME, Pompei P, Ales KL, et al. A new method of classifying prognostic comorbidity in longitudinal studies: development and validation. J Chronic Dis 1987;40:373-83.

25. Vellas $B$, Villars $H$, Abellan $G$, et al. Overview of the MNA®-ITS history and challenges. J Nutr Health Aging 2006;10:456.

26. Rubenstein LZ, Harker JO, Salva A, et al. Screening for Undernutrition in Geriatric Practice: developing the Short-Form MiniNutritional Assessment (MNA-SF). J Gerontol A Biol Sci Med Sci 2001;56:M366-M372.

27. Guigoz $Y$, Jensen $G$, Thomas D, et al. The Mini Nutritional Assessment (MNA®) review of the literature-what does it tell Us?. J Nutr Health Aging 2006;10:466.

28. Mann J, Larsen P, Brinkley J. Exploring the use of negative binomial regression modeling for pediatric peripheral intravenous catheterization. J Med Stat Inform 2014;2:6.

29. StataCorp. Stata 12 Base Reference Manual. College Station, TX: Stata Press, 2011

30. StataCorp. Stata Statistical Software: release 12. College Station, TX 2011.

31. Patient Centered Assessment Method [PCAM] Online [Internet] Stirling and Minnesota: Universities of Stirling and Minnesota, c2013, http://www.pcamonline.org/training-resources.html.

32. Shevlin M, Miles JNV, Davies MNO, et al. Coefficient alpha: a usefu Indicator of reliability? Pers Individ Dif 2000;28:229-37. 